\title{
CORRELATION BETWEEN ENERGY AND VOLUME EXPANSION FOR GRAIN BOUNDARIES IN FCC METALS
}

\author{
D. Wolf \\ Materials Science Division, Argonne National Laboratory, Argonne, Illinois 60439 \\ (Received June 9, 1989) \\ (Revised September 6, 1989)
}

\section{INTRODUCTION}

Three decades ago, Seeger and Schottky ${ }^{\dagger}$ presented a very simple model suggesting that the excess energy, $\gamma$, and excess volume per unit area, $\delta \mathrm{V}$, of grain boundaries (GBs) in metals are closely interrelated. They assumed the GB region, of width $\delta \mathrm{V}$ and surrounded by bulk material, to be characterized by a lower atom and therefore electron density. Considering only the change in the kinetic and potential energies of the electrons, they solved the one-dimensional Schrodinger equation to show that $\gamma=\gamma(\delta \mathrm{V})$ is a practically linear function. An interesting aspect of their model is the fact that the detailed atomic structure does not enter at all, except via the parameter $\delta \mathrm{V}$. More recently, using more sophisticated electronic structure methods, Ferrante and Smith 2 demonstrated that some normalized expansion parameter is also the key parameter in the energies of bimetallic interfaces, and that the detailed atomic structure may be irrelevant in the basic shape of the $\gamma(\delta \mathrm{V})$ binding curve.

That the excess volume is an important structural parameter associated with GBs is also apparent from simple hard-sphere models ${ }^{3}$. Following the destruction of the perfect-crystal stacking due to the introduction of a GB into a stack of lattice planes, the crystal has to expand locally at the interface to accommodate the structural mismatch. In these models it is assumed that the most stable GB structure is the one which yields the smallest excess volume per unit GB area, an assumption verified by the atomistic simulation results presented below.

Experimental evidence, based on the effect of hydrostatic pressure on the relative energies of different $\mathrm{GBs}, 4$ supports these concepts. Rotating sphere-on-a-plate experiments suggest that those GBs which give rise to energy cusps show a smaller excess volume than the higher-energy boundaries outside of these cusps. ${ }^{4}$ More recent high-resolution TEM experiments provide direct evidence for the existence of volume expansions at GBs, 5-7 although their precise magnitude remains difficult to determine. Also, no direct information on the GB energy is available from these observations.

Atomistic computer simulations for metals, employing central-force potentials, have provided little information on volume expansions at GBs because virtually all such simulations were carried out at constant volume. By their very nature, the interactions between metal atoms near an interface are anisotropic because the effective electron density which mediates these interactions depends on direction. This anisotropy, in principle, rules out the use of pair potentials, which were employed in most previous GB simulations. These conceptual problems associated with the use of pair potentials in the simulation of inhomogeneous metal systems have been partially overcome only recently with the development of many-body potentials. 8,9 In these potentials, the local-volume dependence of the binding energy is considered explicitly, via the net electron density experienced by every atom due to its interaction with the surrounding atoms. Conceptually they are therefore based on a much better physical description 
of metallic bonding than are pair potentials. Like pair potentials, however, they are strictly empirical potentials, and one can never be certain that they reproduce the properties of a specific material other than those to which they were fitted. For that reason, in this paper the correlation between $\gamma$ and $\delta \mathrm{V}$ will be investigated for both a many-body and a very simple pair potential. The comparison of the results will provide insight into the role of many-body effects in GBs; also, it allows identification of phenomena which are entirely independent of potential and, hence, representative of generic properties of fcc materials.

\section{COMPUTATIONAL PROCEDURE}

The iterative energy minimization procedure ("lattice statics") employed to relax GBs at zero temperature was described in detail in Ref. 10. In addition, a constant-pressure relaxation method was used which permits the unit-cell volume to increase or decrease in response to the internal pressure in the direction of the GB-plane normal, thus enabling the GB to expand or contract. Also, by computing the forces which the two halves of a bicrystal exert on eachother, translations parallel to the GB plane are permitted.

Both a many-body potential of the Embedded-Atom-Method (EAM) type, fitted to five properties of $A u, 8$ and a simple Lennard-Jones (LJ) pair potential, fitted to two perfect-crystal properties of $\mathrm{Cu}, 11$ will be used. The related cohesive-energy curves for a perfect crystal are shown in Fig. 1. To emphasize the different shapes of the curves associated with the two potentials, the values at the related minima have been subtracted. The effect of these shape differences on the predicted GB energies and volume expansions will be discussed below.

The unrelaxed input geometries of the symmetrical and asymmetrical tilt and twist boundaries investigated below were obtained by misorienting point-defect free perfect-crystal slabs appropriately with respect to each-other. Although reconstruction and impurity segregation may be important in real materials, these simple point-defect free model systems are useful to study structure-property correlations prior to introducing additional complications, such as impurities, reconstruction, temperature, etc.
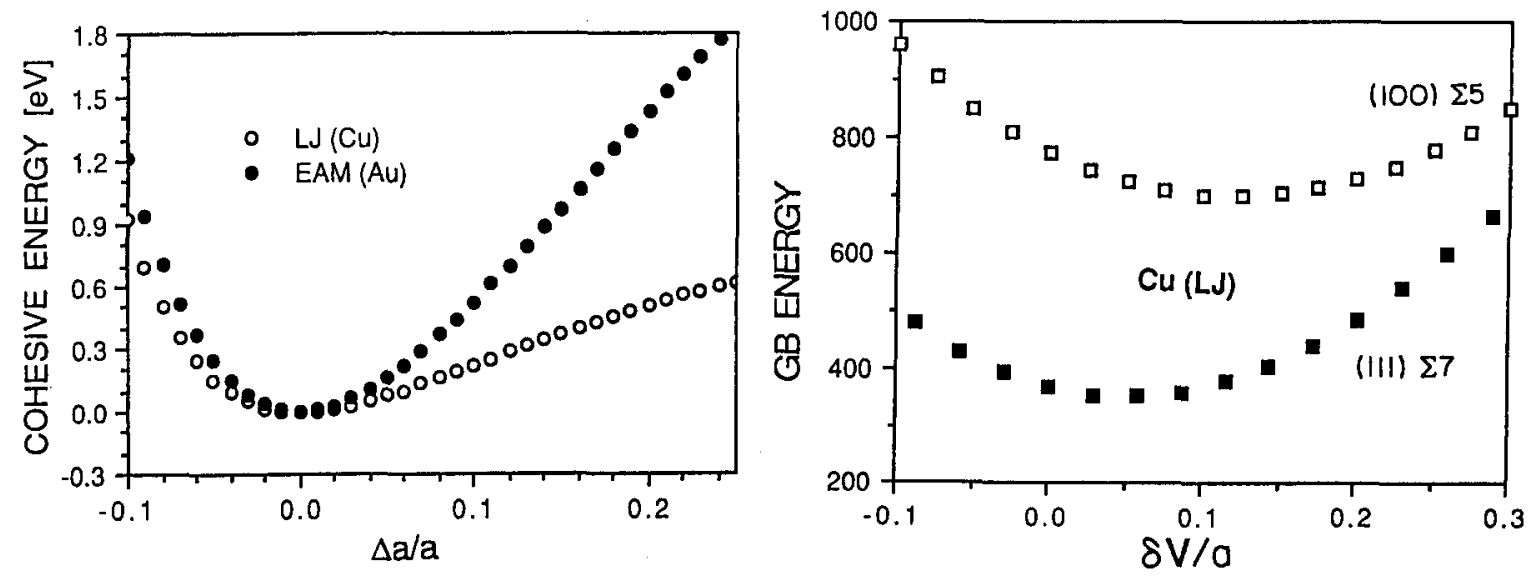

Fig. 1. (left): Cohesive energy vs. lattice parameter for the potentials employed in this paper. The equilibrium cohesive energies of $-3.91 \mathrm{eV}$ and $-1.03 \mathrm{eV}$ for the EAM and LJ potentials, respectively, were subtracted to emphasize the shape differences.

Fig. 2 (riaht): GB energy (in $\mathrm{mJ} / \mathrm{m}^{2}$ ) vs. $\delta \mathrm{V} / \mathrm{a}$ for two twist GBs in the fcc lattice (LJ potential). 


\section{SIMULATION RESULTS}

A simple method for determining the eqilibrium volume expansion per unit area, $\delta \mathrm{V}$, is illustrated in Fig. 2 for the so-called $\Sigma 5$ boundary on the (100) plane and the $\Sigma 7$ boundary on the (111) plane. (Here $\Sigma$ denotes the inverse density of coincident-site lattice (CSL) positions which are in common to the two halves of a bicrystal, provided no translation parallel or perpendicular to the GB plane is permitted.) In these calculations, the positions of the faraway perfect-crystal regions surrounding the GB were held fixed at a constant value of $\delta \mathrm{V}$, and a constant-volume relaxation was performed for each value of $\delta \mathrm{V}$. Similar to Fig. 1 , the $\mathrm{GB}$ energy shows a minimum at the eqilibrium value of $\delta \mathrm{V}$, which is different, however, for the two GBs considered. Interestingly, as suggested by Meiser and Gleiter ${ }^{4}, \delta \mathrm{V}$ is indeed smaller for the boundary with the lower energy. Fig. 2 also illustrates the effect of a restriction to constant volume in the simulation, which leads to systematically larger $\mathrm{GB}$ energies and to substantially different relative energies of the two GBs. This demonstrates how important it is to allow the bicrystal volume to equilibrate. It should be mentioned in this context that the definition of the GB energy as the change in Gibbs free energy, $G$, with $G B$ area, $A$, at constant temperature, $T$, pressure, $p$, and number of particles, $N$, (including composition), according to 12

$$
\gamma=(\partial G / \partial A) T, p, N
$$

dictates constant-pressure simulations. Constant-volume simulations, therefore, lead not only to numerically wrong values of $\gamma$ for a given potential, but energies thus determined are conceptually not the true excess energy in the Gibbsian sense. Consistent with the definition of $\gamma$ in Eq. (1) is the definition of $\delta \mathrm{V}$ (to be distinguished from the overall volume $\mathrm{V}$ ) according to 13

$$
\delta V=(\partial V / \partial A) T, p, N
$$

which indicates that the excess volume of a GB is to be determined under the same conditions as the excess energy.

The method of extracting $\gamma$ and $\delta \mathrm{V}$ from a series of constant-volume calculations is not very efficient. A systematic investigation of the $\gamma(\delta \mathrm{V})$ correlation was therefore performed by using the constant-pressure relaxation procedure described above. The results thus obtained for four different types of boundaries are summarized in Figs. 3(a) and (b).
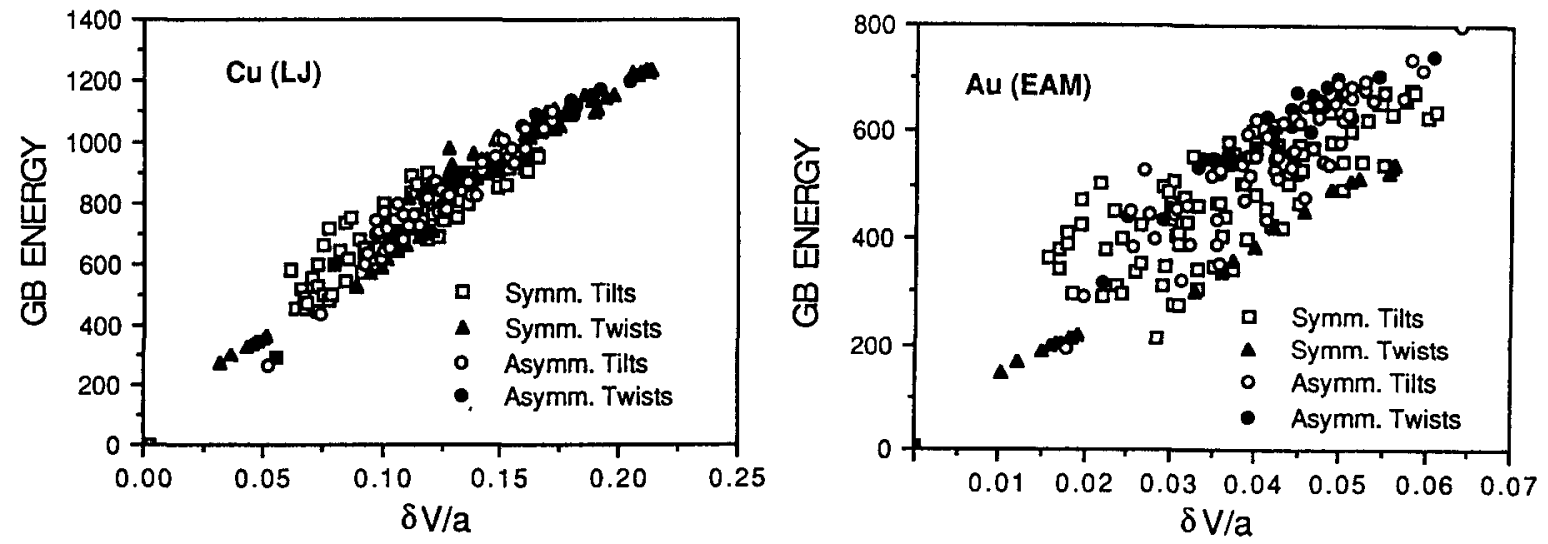

Fig. 3: GB energy vs. volume expansion for the LJ (left) and EAM potential (right). An equally good linear correlation was reported recently by Chen et al. ${ }^{15}$ for STGBs in $\mathrm{Ni}_{3} \mathrm{Al}$. 
Table 1: Parameters of a least-squares fit to the data in Figs. 3(a) and (b), according to $\gamma(\delta \mathrm{V})=$ $\alpha+\beta d V$. Also listed are results (which include symmetrical GBs only) ${ }^{14}$ for an EAM potential for $\mathrm{Cu} . \alpha$ is in units of $\mathrm{mJ} / \mathrm{m}^{2} ; \beta$ is in units of $\mathrm{mJ} /\left(\mathrm{m}^{2} \mathrm{a}\right)$, where $a$ is the ideal-crystal lattice parameter listed also in the Table (in Angstrom units).
Au (EAM)
$\mathrm{Cu}$ (LJ)
$\mathrm{Cu}$ (EAM)

\begin{tabular}{lccc}
\hline$\alpha$ & 105.6 & 120.7 & 104.5 \\
$\beta$ & 1021 & 5497 & 9636 \\
$\mathrm{a}$ & 4.0828 & 3.6160 & 3.6212
\end{tabular}

As is well known, five macroscopic degrees of freedom (dof) are needed to characterize a single bicrystalline GB. The boundaries considered in Fig. 3 range from the simplest symmetrical tilt boundaries (STGBs) with 2 dof, via symmetrical twist GBs (3 dof) and asymmetrical tilt GBs (4 dof), to asymmetrical twist (or general) boundaries (with 5 dof). In total, Figs. 3(a) and (b) each contain 85 STGBs, 74 pure twist GBs, 55 asymmetrical tilt GBs, and 39 general boundaries.

(These simulations are discussed in a recent series of articles). ${ }^{14}$ In view of this variety of GBs considered, the reasonably good linear correlation between $\gamma$ and $\delta V$ in these results, obtained for both potentials, is rather remarkable (see also Table 1). With the values listed in Table 1 one could, in principle, obtain approximate GB energies from volume expansions extracted, for example, from TEM observations.

\section{READ-SHOCKLEY ANALYSIS OF EXCESS VOLUMES}

In a recent article 16 it was suggested that the modified Read-Shockley 17 expression,

$$
\gamma(\theta)=\sin \theta\left[E_{C}-E_{S} \ln (\sin \theta)\right] / b \quad,
$$

may be valid not only for low- but also high-angle boundaries, in which the dislocation cores overlap completely. In Eq. (3), $\theta$ denotes the CSL misorientation angle, while $E_{C} / b$ and $E_{S} / b$ represent the core and strain-field energy per unit length of an isolated lattice dislocation; $b$ is the length of the Burger's vector. Whereas the physical reasons for the validity of Eq. (3) even for high-angle GBs are not clearly understood 16 , strong evidence for its validity, for both
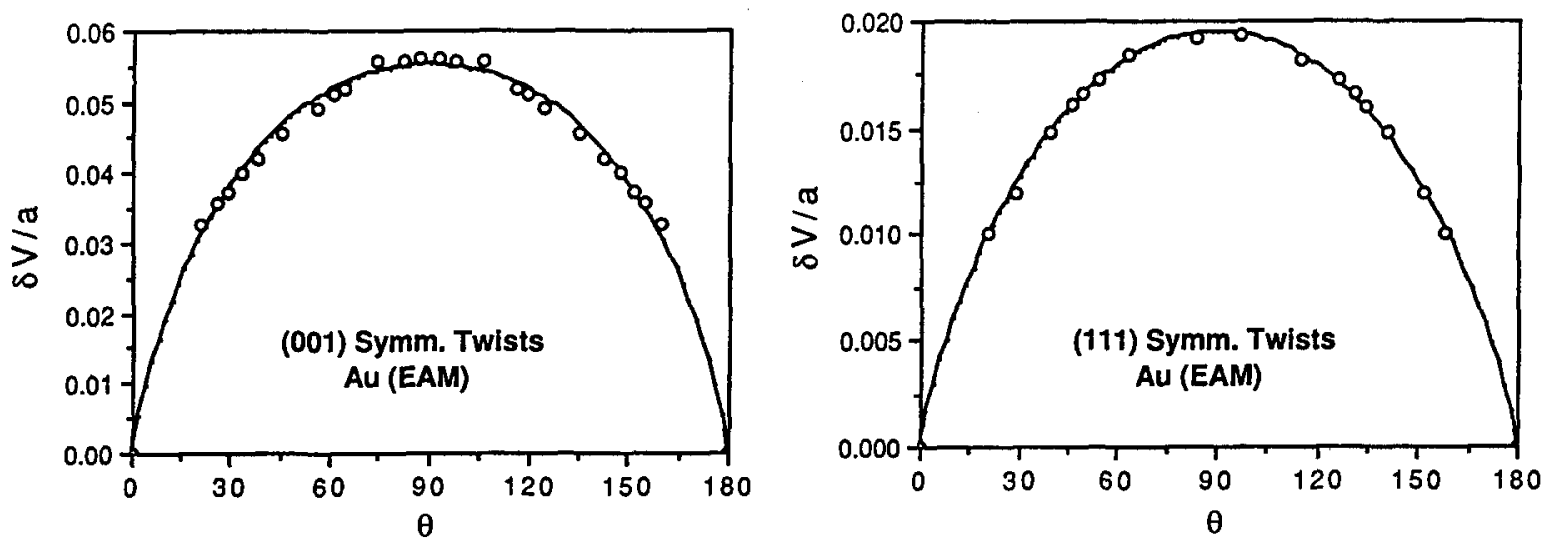

Eig. 4: Excess volume (in units of a) vs. twist angle for (001) (left) and (111) twist GBs in Au (right half). The solid lines represent a least-squares fit of Eq. (4) to the data points. 
symmetrical and asymmetrical boundaries, was presented in Ref. 16 . This evidence was obtained from computer simulations similar to the ones presented here, using the same two interatomic potentials. Owing to the close interrelation between $\gamma$ and $\delta \mathrm{V}$ observed above, one would expect that a relationship analogous to Eq. (3) should exist for the excess volume. Therefore, in analogy to Eq. (3) we write :

$$
\delta V(\theta)=\sin \theta\left[\delta V_{C}-\delta V_{S} \ln (\sin \theta)\right] / b \quad
$$

where $\delta V_{c} / b$ is the excess core volume per unit length of a dislocation, while $\delta V_{S} / b$ is the related strain-field contribution. As in Eq. (3), the range of $\theta$ values for a given misorientation axis has to be normalized so as to cover the full range from 0 to $180^{\circ}$. In cases in which symmetries exist for a certain misorientation axis, $\theta$ therefore may be a multiple of the usual CSL misorientation angle 16 .

For both potentials a least-squares fit of Eq. (4) to the data in Fig. 3 , with $\delta V_{c}$ and $\delta V_{S}$ as adjustable parameters, was performed. Typical results thus obtained are shown in Figs. 4 (for pure twist GBs for the EAM potential) and 5 (for symmetrical tilt and general GBs, simulated via the LJ potential). Analogous to the results for the GB energy 16 , Figs. 4 and 5 suggest that the Read-Shockley model works equally well in predicting the excess volumes of high-angle boundaries, based entirely on the volume changes associated with low-angle GBs, i.e., of isolated lattice dislocations. As in case of the energies, the fits in Figs. 4 and 5 do not include the minor cusps which appear whenever a GB with a particularly small planar unit-cell area, $A=\Gamma A_{1}$, is approached (where $A_{1}$ is the smallest possible unit-cell area for a given misorientation, and $\Gamma$ is the density of planar CSL sites) ${ }^{14}$.
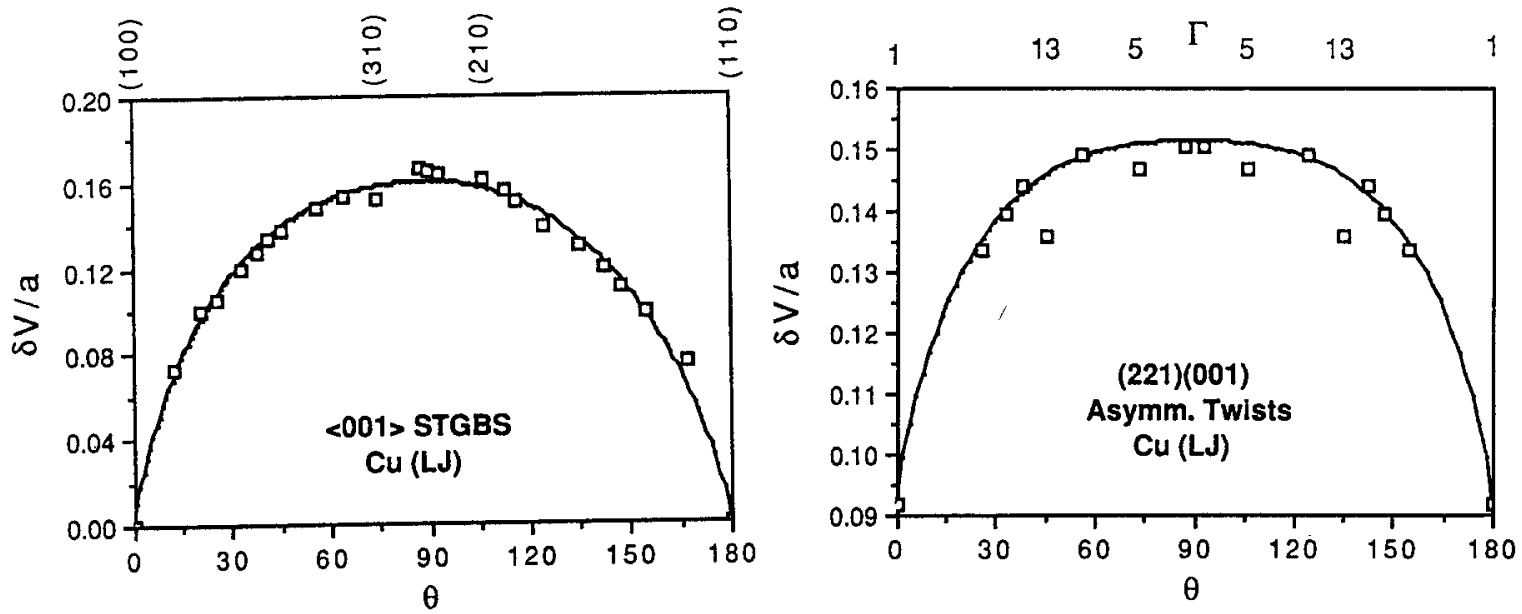

Fia. 5: Excess volume, $\delta \mathrm{V} / \mathrm{a}$, vs. misorientation angle for $<001>\mathrm{STGBs}$ (left) and (221)(001) asymmetrical twist boundaries. Solid lines represent a least-squares fit of Eq. (4) to the data points.

\section{CONCLUSIONS}

We note that, according to Figs. $3(a)$ and (b), the volume expansions and energies obtained for the LJ potential are generally much larger than for the EAM potential. The reason for this difference is readily apparent from Fig. 1, according to which an increase in volume is much less costly in energy for the LJ than for the EAM potential. A similar difference to the LJ results was also obtained for the EAM potential fitted for $\mathrm{Cu}^{14}$. This illustrates that, although 
behavior, significant quantitative differences may be obtained for different potentials fitted to the same material. Also, in numerous cases rather different optimum translational states parallel to the GB plane are obtained for the two potentials (for details see Ref. 14), as one would expect according to Ref 18. Although EAM potentials represent a given material better than the simple LJ potential (because they were fitted to a larger number of empirical properties), for lack of quantitative experimental information the above results cannot be substantiated. However, since two potentials as diverse as the LJ and EAM potentials yield the same generic behavior, we are lead to conclude that:

(a) The practically linear $\gamma(\delta \mathrm{V})$ correlation, the interpretation of which was first suggested by Seeger and Schottky, 1 is a generic property of GBs in fcc metals; this correlation, in principle, permits GB energies to be extracted from TEM measurements.

(b) Local-volume effects, considered only in the EAM potential, affect the magnitude of the GB energy and volume expansion but not the basic behavior obtained from the simulations.

(c) The detailed atomic structure, including translations parallel to the GB plane, found to be rather different for the two potentials in numerous cases, has little effect on the generic $\gamma(\delta \mathrm{V})$ correlation reported here.

We also note that, according to Figs. 4 and 5, the volume expansion at high-angle GBs in the Read-Shockley sense (in which the dislocation cores overlap substantially) is very well described in terms of Eq. (4), the underlying physics of which is based entirely on the ReadShockley picture for low-angle boundaries; i.e., on the physics of isolated lattice dislocations. We hope that this puzzling observation will contribute to a renewed discussion of the role of lattice dislocations in the basic physics of high-angle GBs.

Acknowledgment. This work was supported by the U. S. Department of Energy, BES Materials Sciences, under Contract No. W-31-109-Eng-38.

\section{REFERENCES}

1. A. Seeger and G. Schottky, Acta Metall. 7, 495 (1959).

2. J. Ferrante and J. R. Smith, Phys. Rev. B 31, 3427 (1985).

3. See, e.g., H. J. Frost, M. F. Ashby and F. Spaepen, Scripta Metall. 14, 1051 (1980).

4. H. Meiser and H. Gleiter, Scripta Metall. 14, 95 (1980).

5. G. J. Wood, W. M. Stobbs and D. J. Smith, Phil. Mag. A 50, 375 (1984).

6. K. L. Merkle and D. J. Smith, Phys. Rev. Lett. 59, 2887 (1987).

7. K. L. Merkle, Scripta Metall. (submitted).

8. M. S. Daw and M. I. Baskes, Phys. Rev. B 33,7983 (1986).

9. M. W. Finnis and J. E. Sinclair, Phil. Mag. A 50, 45 (1984).

10. D. Wolf, Acta Metall. 32, 245, ibid. 735 (1984), and J. Am. Cer. Soc. 67, 1 (1984).

11. J. F. Lutsko, D. Wolf, S. Yip, S. R. Phillpot and T. Nguyen, Phys. Rev. B 38, 11572 (1988).

12. See, for example, W. D. Kingery, H. K. Bowen and D. R. Uhlmann, Introduction to Ceramics (2nd edition), Wiley, New York (1976).

13. H. B. Aaron and Bolling, in Grain Boundary Structure and Properties, G. A. Chadwick and D. A. Smith (eds.), Academic Press, New York, 1976, p.107.

14 D. Wolf, Scripta Metall. 23, 377 (1989), Acta Metall. 37, 1983 (1989), and ibid. (in press).

15. S. P. Chen, D. Srolovitz and A. F. Voter, J. Mater. Res. 4, 62 (1989).

16. D. Wolf, Scripta Metall. (in press).

17. W. T. Read and W. Shockley, Phys. Rev. 78, 275 (1950).

18. D. Wolf, J. Phys. Colloque C4 46, C4-197 (1985). 